\title{
METHODOLOGY AND NECESSARY EQUIPMENT TO MITIGATE CAPACITY LIMITATIONS CAUSED BY COVID-19 IN TEACHING LABORATORIES
}

\author{
E. Peñalvo-López, V. León-Martínez, J. Montañana-Romeu, F.J. Cárcel-Carrasco \\ Universitat Politècnica de València (SPAIN)
}

\begin{abstract}
The reduction of the space available for students in the classrooms of teaching laboratories, due to the pandemic caused by the SARS-CoV-2, requires the provision of the laboratories with additional equipment, as well as the application of procedures or protocols that guarantee that all students receive practical education with levels of quality similar to those existing in face-to-face classes, prior to the arrival of the coronavirus. This paper describes the methodology and resources used to teach practical classes in one of the Electrical Engineering teaching laboratories, at the Polytechnic University of Valencia. The use of web cameras, complemented with the use of the Microsoft Teams platform, has allowed all students actively attend laboratory classes in real time, within the established schedule, some in person and others in online mode.
\end{abstract}

Keywords: Electrical labs, SARS-CoV-2, practical teaching, hybrid learning.

\section{INTRODUCTION}

The Universitat Politècnica de València has promoted the teaching with the greatest possible students' presence, considering the capacity limitations in teaching classrooms, established by the Action Protocol for the 2020-21 academic course of the Valencian Community [1] and the Prevention Plan of the Polytechnic University of Valencia [2], because of COVID-19. The model chosen for teaching the classes with a number of students that exceed the maximum capacity of the classroom is the hybrid, which is not exactly the blended model, studied in references [3-8], but is closer to the called "two classrooms at the same time" in reference [9]. In the hybrid model, there are students attending classes of theory in person, with the security measures and resources indicated in reference [10], in a number not exceeding the maximum capacity set by the Protocols [1, 2]. Meanwhile, the rest of the students receive these classes at their homes, in real time, through the Microsoft Teams platform, thanks to a video camera service focused on the blackboards, in each classroom. These nonclassroom students can communicate with the teacher, making comments and asking questions at any time, just like classroom students, thereby avoiding the inconveniences derived from the teacher's lack of dedication towards non-classroom students, indicated in [9].

The hybrid teaching implemented at the Polytechnic University of Valencia is, in our opinion, the teaching-learning model with levels of quality closer to face-to-face teaching, even when there is a loss of fluency in gestural communication, especially of the non-classroom students, so necessary so that the teacher can give feedback on their teaching classes. However, with hybrid teaching, nonclassroom students are in a position to receive the same knowledge and acquire the same skills as classroom students.

The application of hybrid teaching has an important limitation when teaching the laboratory classes, even with the same resources (video cameras focused on the blackboard and the Teams platform) as the theory classes, since the laboratory classes require a greater and more active participation of students. However, laboratory classrooms have been the most affected in terms of capacity reduction, after the implementation of minimum safety distances in teaching classrooms, recommended by the Protocols $[1,2]$.

Section 4.1.2. of the UPV Prevention Plan [2], corresponding to the measures applicable to teaching classrooms, establishes that "the capacity of these spaces will be restructured taking into account a minimum safety distance between students of 1.5 meters and, at least, 2 meters between teachers and students". Also, section 4.1.4. of the aforementioned Prevention Plan [2] indicates that, "in general, capacity should be limited to one person for every $5 \mathrm{~m}^{2}$ in teaching laboratories where mobility of students is necessary during classes", maintaining interpersonal distance 1.5 meters in teaching laboratories where classes are taught in static mode. 
The application of the above-mentioned sections of the Prevention Plan [2] to the spaces of the laboratory classrooms of the Circuits Teaching Unit of the Department of Electrical Engineering of the Polytechnic University of Valencia, has led to a decrease in the available capacity to a third of the existing capacity before the pandemic. Consequently, the presence of each student in the laboratory classes has also been reduced to one third.

In this scenario, there are significant difficulties in teaching hybrid laboratory classes, since nonclassroom students (two out of three in each class) could only participate actively in one third of these classes, even with the resources of the video camera focused on the blackboard and the use of the Teams platform. Nor would it be desirable to reduce the number of practical classes to a third of the total and repeat each practice three times, so that all students attend the same practices, since students would not receive all the competencies contained in the Teaching Guide of each subject.

This paper presents a solution to the problem of teaching hybrid laboratory classes, with limited capacity for teaching laboratories. This solution consists of installing a video camera on each of the tables or workstations in the laboratory, as well as a computer that incorporates the Teams platform. Each table or laboratory station will be assigned a group of three students, at most, and only one of them will attend each practical session in person, taking into account the limitation of the laboratory capacity indicated by the Protocols [1,2]. The other two students can actively participate in the development of the practice from their homes, since they would have a direct view of the laboratory station and could communicate in real time with the classroom student through the Teams platform. The classroom students take turns in each practice session, so that all of them have attended the same number of laboratory practices.

This article describes a pilot experience of teaching hybrid laboratory classes to one of the teaching laboratory classrooms of the Department of Electrical Engineering of the Universitat Politècnica de València, with the application of the indicated above resources. Likewise, the protocol developed for the teaching hybrid laboratory classes is presented, as well as the problems that have arisen in its application and the proposed solutions to overcome them are described.

\section{CONTEXT}

The Circuits Unit of the Department of Electrical Engineering of the Universitat Politècnica de València attends students of 23 subjects from four University degrees and three University Master's degrees from the Higher Technical School of Design Engineering, with an area assigned to teaching laboratories equal to $247 \mathrm{~m}^{2}$. In order to avoid overlaps between the practical classes of the different subjects, this area is not occupied by a single laboratory, but has been divided into four separate spaces. One of these spaces, called Laboratory 1 , has been used to carry out the pilot test of hybrid laboratory classes. This laboratory classroom is shown in Figure 1. It has a useful area of $54.79 \mathrm{~m}^{2}$, with nine workstations, with a maximum capacity of 24 students. In this classroom the practices corresponding to a total of 9 subjects are taught, belonging to the Study Plans of the Master of Aeronautical Engineering and Industrial Engineering and to the Degrees in Aerospace Engineering, in Mechanical Engineering and in Industrial Electronic and Automatic Engineering. At the back of this classroom, there is an office for the laboratory technician as well as a small store and atelier space (Figure 1).

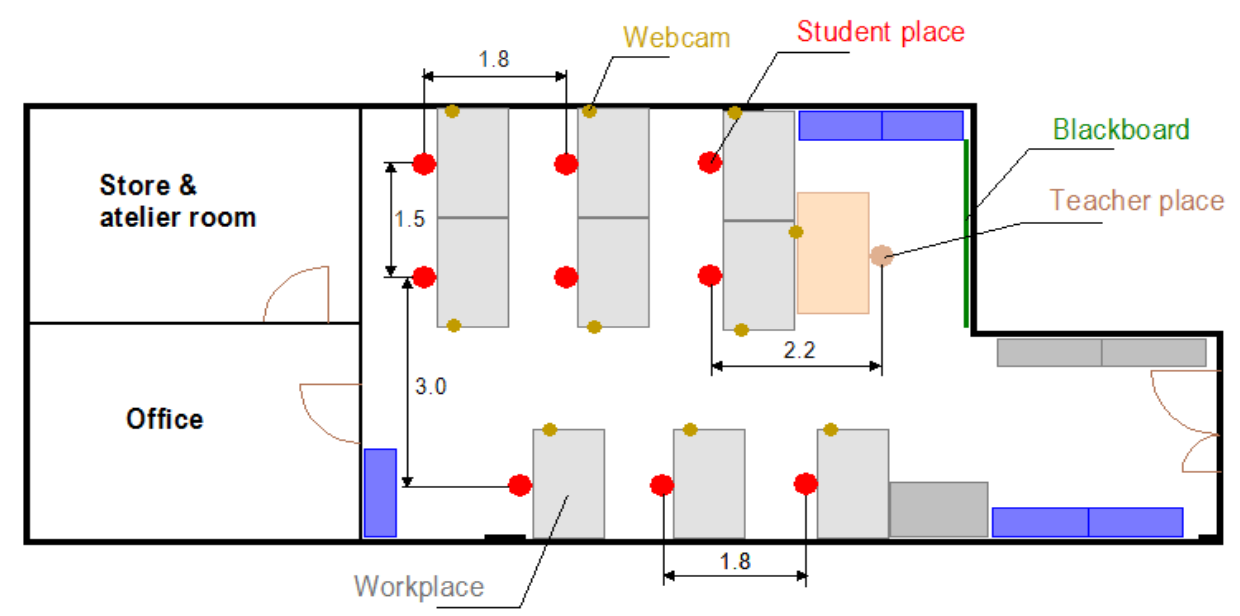

Figure 1. Distribution of elements and workstations in Laboratory 1. (Separation distances are in meters). 
The application of sections 4.1.2. and 4.1.4. of the UPV Prevention Plan [2] has meant a reduction of the laboratory's capacity to one student per workstation, in each practical session, that is, practically a third of the maximum capacity.

A possible solution to save the decrease in available capacity in Laboratory 1 was to reduce the number of laboratory practices taught during the current academic year 2020-21. Given that the Teaching Organization Plan assigns a weekly practical class to each group of practices of the different subjects and that the available capacity of the laboratories has been reduced to a third of the maximum capacity, this solution would force to repeat each practical session over three weeks, so that all students receive the same practical classes. Consequently, with this solution, the number of practices carried out would be reduced to a third and the students would not have reached the competences assigned in the Teaching Guide.

Another solution would have been to carry out all the laboratory practices included in the Teaching Guide for the situation of total presence prior to COVID-19, giving a practice each week to a reduced number of students, in a number equal to a third of the maximum capacity, in compliance with the guidelines of the Action Protocol $[1,2]$. However, this solution has the same drawbacks as the previous one, in terms of the competencies that the students would no longer achieve, since each student would only carry out one in three practices.

The third solution consisted of installing a video camera connected to a computer for each workstation (Figure 1). These cameras are fixed to a flexible support, which can be oriented by the students, allowing a focus on the entire table and the elements available at each workstation. The number of classroom students is one for each workstation (one third of the maximum capacity of the classroom), but non-classroom students can follow the explanations on the teacher's blackboard and interact in developing the practice with the student present in classroom through the video camera installed in each workstation and the Microsoft Teams platform.

The practical classes in Laboratory 1 has been carried out according to this last solution, as it allows all students to be in a position to acquire the competencies that the laboratory classes grant them. These classes are hybrid face-to-face.

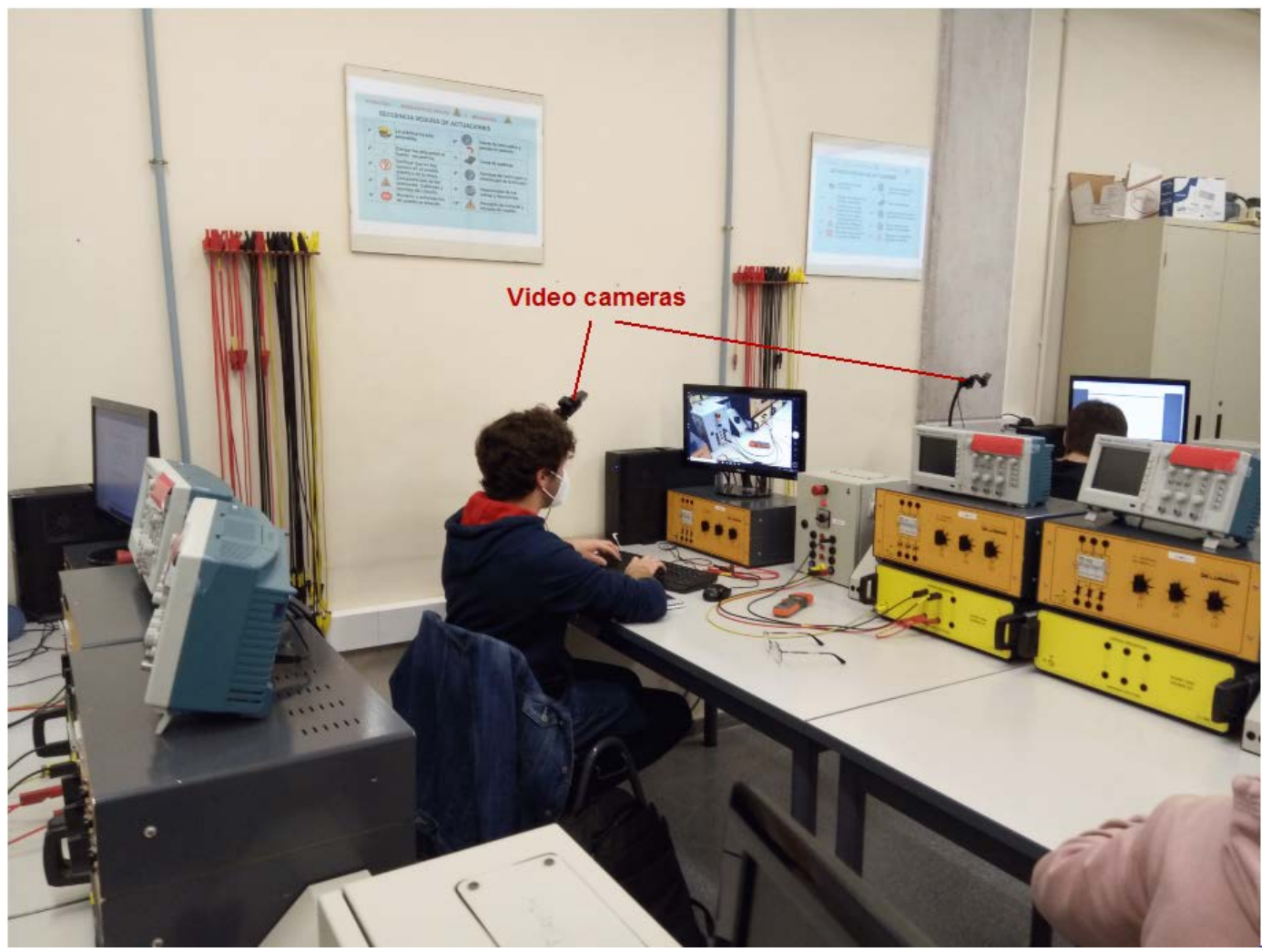

Figure 2. Look of a hybrid laboratory classroom. 
Figure 2 shows a photograph of the classroom during a hybrid lab class. On the computer screen of one of the students, the vision of the workstation that the video camera offers to the non-face-to-face students is observed. Likewise, the photograph shows the arrangement of the video cameras, with their supports, at the workplace.

\section{METHODOLOGY FOR IMPLEMENTING THE LABORATORY HYBRID CLASSES}

The attainment of the hybrid laboratory classes, in the pilot experience, has been adjusted to the following methodology:

1 Creation of as many subgroups of Teams as students are in each group of laboratory practices divided by 3 . The students that make up each subgroup are assigned and the dates on which each student must attend the laboratory class are reported.

2 Before the start of each class, the teacher must ensure that the students in the classroom maintain safety distances before and after entering the classroom. Likewise, the teacher indicates to these students the need to use the hydroalcoholic gel and to clean the workplace before and after the end of the class.

3 Once all the face-to-face students are in the classroom, in their corresponding work stations, the teacher begins the class using the general group of Teams from that group of practices, to:

3-a) list all the students in the group, both face-to-face and not face-to-face; the latter through the Teams platform, and

3-b) briefly explain the theoretical foundations and the measures and requirements to be fulfilled in practice. The teacher can use the video camera that focuses on the blackboard as a support to carry out this action.

4 Carrying out the practice by the students, using the existing video cameras in each workplace and the communication between face-to-face and non-face-to-face students provided by the Teams platform for each of the student subgroups created in the first step of the methodology. For it:

4-a) The student of each subgroup, who attends the class in person in each laboratory workstation, must connect with the non-face-to-face, through their corresponding subgroup of Teams and share with them the images from the video camera installed at your workstation.

4-b) Each face-to-face student will be in charge of making the different assemblies required in practice, using the devices and elements available in his workplace. The non-face-to-face students can supervise the assembly and intervene in the development of the practice, communicating with the face-to face student.

4-c) The face-to-face student must provide his subgroup non-face-to-face colleagues a view of the readings from the measuring devices, so that those can carry out the tasks proposed for each practice. For this, the face-to-face student has the possibility of adjusting the focus and field of view offered by the video camera, which is installed on adjustable supports.

5 In the middle of each practical class, a task will open in PoliformaT, previously created by the teacher, whose closing time coincides with the end of the class. Non-face-to-face students before the end of the class must complete this task, while the face-to-face student can suggest answers to the proposed task, through Teams. With this, it is guaranteed that non-face-to-face students actively participate and are attentive to the development of the practice.

Once the task is delivered, the classroom student and non-face-to-face students of each subgroup can leave the class. Before leaving, the face-to-face student must take care of disconnecting the assemblies made and disinfecting the workstation. 


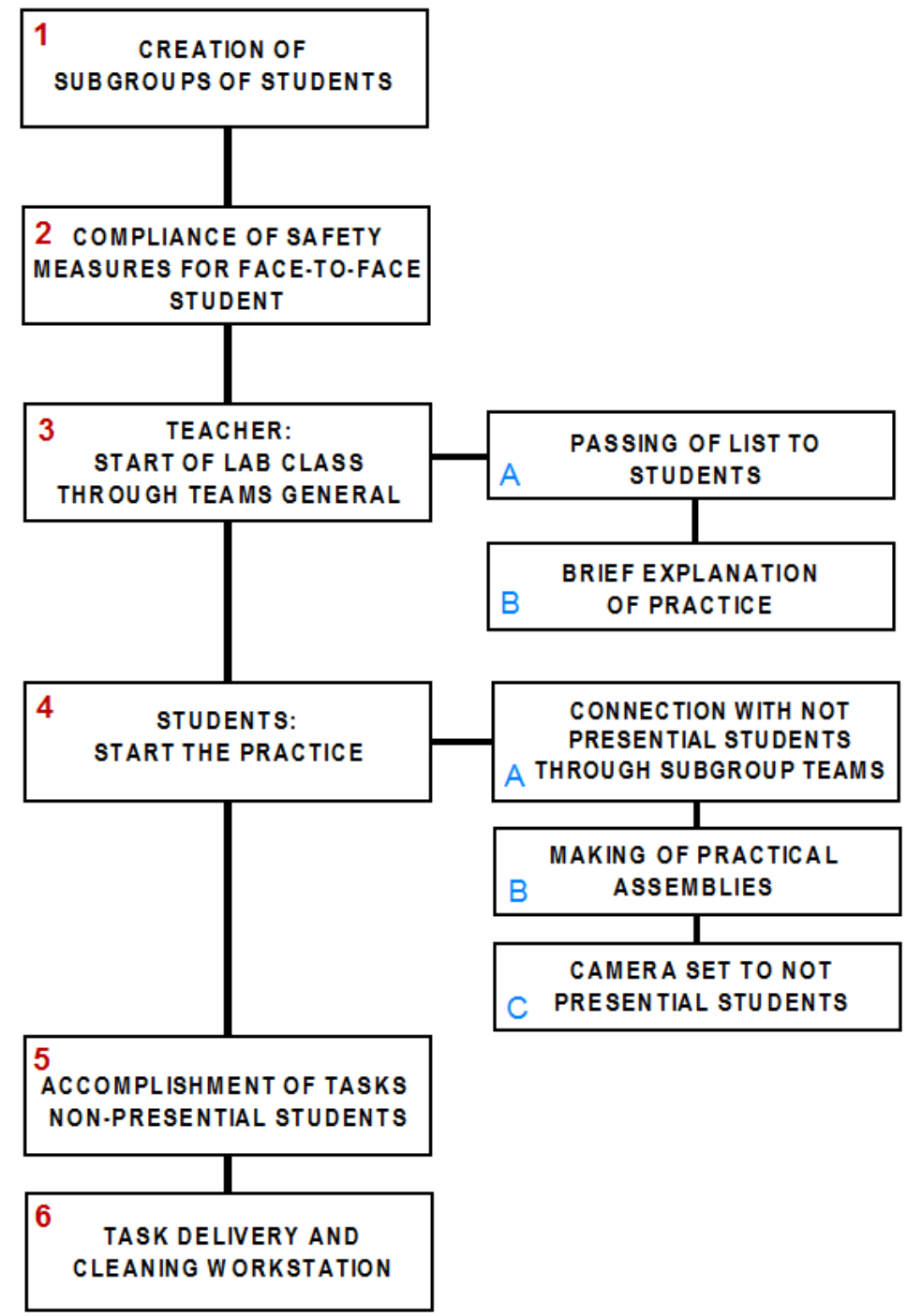

Figure 3. Organization chart of the methodology of the laboratory hybrid classes.

\section{CONCLUSIONS}

The teaching of theory and laboratory classes has been greatly affected by the pandemic caused by the SARS-CoV2, by the closure of teaching centers, first, and by the capacity restrictions of the classrooms, after the opening of teaching centers. The capacity restrictions of the laboratory rooms have been especially important. This, together with the particular requirements of the laboratory classes, in which presence is necessary to achieve the full competencies that these classes confer, has led to a loss of the competencies acquired by the students.

The use of video cameras in each of the workstations in one of the laboratories of the Department of Electrical Engineering of the Universitat Politècnica de València, together with the methodology used to teach the laboratory classes, described in section 3 of this article, have considerably mitigated the negative effects of capacity restrictions. Specifically:

- Each student attends all the laboratory practical classes, some in person and others online.

- The non-face-to-face students in each practice participate actively in the classes, actively participating in the development of the practice.

- In order to avoid that non-face-to-face students in each practice have a passive intervention in class, these students are in charge of completing the tasks proposed for each practice, which must be presented before the end of each class.

- Students in hybrid laboratory classes acquire between 90 and $95 \%$ of the competencies assigned to laboratory practical classes. 


\section{ACKNOWLEDGEMENTS}

Authors will like to thank the Universitat Politècnica de València for the institutional support in the development of this work.

\section{REFERENCES}

[1] Action protocol for the beginning of the 2020-21 University Course (in Spanish). Generalitat Valenciana, Universitat de València, Universitat Politècnica de València, Universitat d'Alacant, Universitat Jaume I, Universitas Miguel Hernández. September 8, 2020.

[2] UPV Prevention Plan. General guidelines of educational entities (in Spanish). Servei Integrat de Prevenció y Salut Laboral of the Universitat Politècnica de València. 08/25/2020.

[3] Gámiz- Sánchez, V. \& Gallego-Arrufat, M.J. Model for analyzing blended learning in Higher Education (in Spanish). Educación XX1, 19(1), 39-61. 2016. doi:10.5944/educXX1.13946

[4] Francis, R. y Shannon, S.J. Engaging with blended learning to improve students' learning outcomes. European Journal of Engineering Education, 38(4), 1-11. 2013.

[5] Area Moreira, M. Training and learning in virtual environments. Potentialities, weaknesses and trends (in Spanish). Crítica, Year 62, № 982, 2012, pp. 33-36.

[6] Moya-Ballester, J. An experience in blended teaching (in Spanish). IX Conference on Research Networks in University Teaching: design of good teaching practices in the current context. Universitat d'Alacant. June 16-17, 2011.

[7] Emerson, L. y MacKay, B. A comparison between paper-based and online learning in higher education. British Journal of Educational Technology, 42(5), 727-735. 2011. doi: 10.1111/j.14678535.2010.01081.x

[8] López-Pérez, M.V., Pérez-López, M.C. y Rodríguez-Ariza, L. Blended learning in higher education: Students' perceptions and their relation to outcomes. Computers \& Education, 56, 818- 826. 2011. doi: 10.1016/j.compedu.2010.10.023

[9] de Santiago, M. Not even the best virtual platform replaces the presence of a teacher in a classroom. Reflections on the blended teaching experience (in Spanish). Blog of the Faculty of Law. November 3, 2020.

[10] Academic criteria for planning the 2020-2021 academic year at the UPV (in Spanish). ViceRector's Office for Studies, Quality and Accreditation. Valencia, July 23, 2020. 